\title{
Cuidados de enfermagem ao paciente renal crônico com cardiopatia em hemodiálise
}

\author{
Nursing care for chronic renal patients with heart disease on hemodialysis \\ Atención de enfermería al paciente renal crónico con cardiopatía en hemodiálisis
}

Thais Oliveira de Paula Lima

ORCID: https://orcid.org/0000-0002-0706-1098 Associação Brasileira de Enfermeiros Cientistas, Brasil E-mail: thaisoplima@gmail.com

Priscila Gramata da Silva Vitorino

ORCID: https://orcid.org/0000-0002-1201-6945 Associação Brasileira de Enfermeiros Cientistas, Brasi E-mail: prigramaenf@yahoo.com.br

Judith Victoria Castillo Mejía

ORCID: https://orcid.org/0000-0001-5257-6878 Associação Brasileira de Enfermeiros Cientistas, Brasil E-mail: iker07castillo@live.com

Natasha Vila Chã

ORCID: https://orcid.org/0000-0002-9781-454X Associação Brasileira de Enfermeiros Cientistas, Brasil E-mail: natashavilacha@ hotmail.com

Victor Hugo de Paula Flauzino

ORCID: https://orcid.org/0000-0001-5156-0030 Associação Brasileira de Enfermeiros Cientistas, Brasil

E-mail: prof.victorflauzino@unyleya.edu.br

Thays Vieira Cusato

ORCID: https://orcid.org/0000-0002-3766-6530 Associação Brasileira de Enfermeiros Cientistas, Brasil

E-mail: thays.vieira.cusato@gmail.com

Daiana Moreira Gomes

ORCID: https://orcid.org/0000-0001-9387-0619 Associação Brasileira de Enfermeiros Cientistas, Brasil E-mail: daigomes_87@hotmail.com

Daniele Vignoli Ribeiro

ORCID: https://orcid.org/0000-0001-5296-8302 Associação Brasileira de Enfermeiros Cientistas, Brasil E-mail: vignoliribeiro@gmail.com

Luana de Oliveira Hernandes ORCID: http://orcid.org/0000-0001-8828-7146 Associação Brasileira de Enfermeiros Cientistas, Brasil

E-mail: luanaoliveira2306@gmail.com

Jonas Magno dos Santos Cesário

ORCID: https://orcid.org/0000-0003-1785-3555 Associação Brasileira de Enfermeiros Cientistas, Brasil

E-mail: prof.jonasmagno@unyleya.edu.br

\begin{abstract}
Resumo
O número de portadores de doenças crônicas aumentou consideravelmente nos últimos anos e isso está relacionado ao envelhecimento da população. O desenvolvimento das doenças renais crônicas, traz consigo complicações cardiovasculares, o que representa um grande desafio para os profissionais de enfermagem ao desenvolver os cuidados necessários. Dessa forma, este estudo buscou identificar os cuidados de enfermagem ao paciente renal crônico com cardiopatia durante o procedimento de hemodiálise convencional. Trata-se de um estudo de revisão bibliográfica de abordagem descritiva e qualitativa. O cuidado de enfermagem ao paciente renal crônico com cardiopatia em hemodiálise requer o desenvolvimento de práticas específicas que atenda as suas reais necessidades. Apesar do grande desafio encontrado pelos enfermeiros em realizar os cuidados necessários em relação à pacientes dialíticos com cardiopatia, manter o acesso vascular, educação em saúde, segurança, mobilização e dieta, são os cuidados mais relevantes considerados pelos enfermeiros, para esses pacientes. $\mathrm{O}$ enfermeiro precisa oferecer um cuidado padronizado, de forma que melhor organize os serviços de enfermagem, além de, facilitar e profissionalizar ainda mais a linguagem utilizada na área. Portanto, o aprimoramento das práticas de enfermagem, assim como, a conscientização para da importância da correta aplicação do processo de enfermagem é de fundamental importância, pois colabora também para que haja a garantia de uma prática de qualidade e segura.
\end{abstract}


Palavras-chave: Doença renal crônica; Hemodiálise; Doenças cardiovasculares; Cuidados de enfermagem.

\begin{abstract}
The number of people with chronic diseases has increased considerably in recent years and this is related to the aging of the population. The development of chronic kidney diseases brings cardiovascular complications, which represents a great challenge for nursing professionals to develop the necessary care. Thus, this study sought to identify the nursing care provided to chronic renal patients with heart disease during the conventional hemodialysis procedure. This is a literature review study with a descriptive and qualitative approach. Nursing care for chronic renal patients with heart disease undergoing hemodialysis requires the development of specific practices that meet their real needs. Despite the great challenge faced by nurses in providing the necessary care for dialysis patients with heart disease, maintaining vascular access, health education, safety, mobilization, and diet are the most relevant care considered by nurses for these patients. Nurses need to offer standardized care, to better organize nursing services, in addition to facilitating and further professionalizing the language used in the area. Therefore, the improvement of nursing practices, as well as the awareness of the importance of the correct application of the nursing process is of fundamental importance, as it also contributes to ensuring a quality and safe practice.
\end{abstract}

Keywords: Chronic kidney disease; Hemodialysis; Cardiovascular diseases; Nursing care.

\title{
Resumen
}

El número de personas con enfermedades crónicas ha aumentado considerablemente en los últimos años y esto está relacionado con el envejecimiento de la población. El desarrollo de enfermedades renales crónicas trae consigo complicaciones cardiovasculares, lo que representa un gran desafío para los profesionales de enfermería a la hora de desarrollar los cuidados necesarios. Así, este estudio buscó identificar los cuidados de enfermería brindados a los pacientes renales crónicos con cardiopatía durante el procedimiento de hemodiálisis convencional. Se trata de un estudio de revisión de la literatura con enfoque descriptivo y cualitativo. La atención de enfermería al paciente renal crónico con cardiopatía en hemodiálisis requiere el desarrollo de prácticas específicas que satisfagan sus necesidades reales. A pesar del gran desafío que enfrentan las enfermeras para brindar la atención necesaria a los pacientes en diálisis con cardiopatías, el mantenimiento del acceso vascular, la educación en salud, la seguridad, la movilización y la alimentación son los cuidados más relevantes que consideran las enfermeras para estos pacientes. El enfermero necesita ofrecer una atención estandarizada, con el fin de organizar mejor los servicios de enfermería, además de facilitar y profesionalizar aún más el lenguaje utilizado en el área. Por tanto, la mejora de las prácticas de enfermería, así como la conciencia de la importancia de la correcta aplicación del proceso de enfermería es de fundamental importancia, ya que también contribuye a asegurar una práctica de calidad y segura.

Palabras clave: Enfermedad renal crónica; Hemodiálisis; Enfermedades cardiovasculares; Cuidados de enfermería.

\section{Introdução}

O número de portadores de doenças crônicas aumentou consideravelmente nos últimos anos e isso está relacionado ao envelhecimento da população. A população idosa é a faixa etária mais atingida por doenças crônicas e entre os principais problemas, pode-se citar doença renal crônica, oriunda, em sua maioria, de comorbidades como diabetes e hipertensão arterial. O desenvolvimento das doenças renais crônicas, traz consigo complicações cardiovasculares, o que representa um grande desafio para os profissionais de enfermagem ao desenvolver os cuidados necessários. Os enfermeiros enfrentam também o desafio de como prevenir o desenvolvimento de complicações em pacientes acometidos por essas doenças (Ribeiro et al., 2020).

De acordo com a Sociedade Brasileira de Nefrologia (SBN, 2020) é considerado como doença renal crônica (DRC) toda lesão que afeta os rins e persiste por três meses ou mais e o seu estágio "final" é mais conhecido como insuficiência renal crônica (IRC). A DRC é uma doença caracterizada pela dificuldade dos rins em exercer seu papel comum de eliminar as substâncias indesejadas do corpo por meio da urina, o que causa o acúmulo de substâncias tóxicas, como por exemplo, produtos de descarte como a ureia e a creatinina e isso pode contribuir para o desequilíbrio fisiológico, pois os rins não conseguem realizar a filtração do sangre de maneira eficaz. Com o objetivo de corrigir esse desequilíbrio, a hemodiálise é a alternativa utilizada para o tratamento de pacientes acometidos pela DRC, pois realiza a filtração sanguínea por meio da utilização de máquinas que fazem o papel de rins artificiais (Matos, 2020). 
Segundo o censo da SBN, o Brasil está entre os principais países que representam os maiores números de casos de pacientes dialíticos do mundo que faze o uso de hemodiálise, com o Japão e os Estados Unidos no topo do ranking da pesquisa. Em 2018, o Brasil apresentou um número de casos três vezes maior de pacientes dialíticos em relação ao ano 2000. Esses números, passaram de 42.695 em 2000, para mais de 133 mil casos de pacientes em 2018. Os estudos mostram ainda que o número de morte aumenta gradativamente no país devido as complicações do quadro da doença, assim como à falta de padronização na assistência e à falta de incorporação tecnológica (Neves et al., 2020).

O tratamento por meio da hemodiálise não ocasiona a cura da DRC, mas reduz o agravamento da doença. São muitos problemas que os pacientes acometidos pela DRC podem enfrentar ao fazer uso da hemodiálise e, uma das principais causas de mortalidade, é o desenvolvimento de doenças cardiovasculares em pacientes dialíticos. O acesso vascular possui extrema importância para os pacientes que fazem uso de hemodiálise, pois como este acesso está diretamente ligado ao órgão responsável pelo bombeamento sanguíneo do corpo, que é o coração, se faz necessário o contínuo monitoramento desse paciente antes, durante e após a realização do tratamento. $\mathrm{O}$ uso contínuo da hemodiálise pode desencadear cardiopatia isquêmica - por uma crítica obstrução das coronárias, alterações microvasculares e alterações morfofuncionais do ventrículo esquerdo (VE), em resposta à sobrecarga de pressão e volume, ambas relacionadas ao alto fluxo do sangue, principalmente em pacientes hipertensos que fazem uso de anti-hipertensivos, pois a hemodiálise diminui o débito cardíaco e a resistência vascular periférica e pode causar uma hipotensão súbita (Silva et al., 2020)

Nesse contexto, o enfermeiro é o profissional que possui maior contato com o paciente e deve estar sempre alerta para possíveis intercorrências durante a hemodiálise e deve controlar os riscos relacionados ao procedimento. É necessário que o enfermeiro desenvolva, durante o processo de cuidados de enfermagem, o monitoramento constante durante a sessão de hemodiálise e assim, garantir a segurança deste paciente (Santos et al., 2021).

Assim como o enfermeiro deve ser capaz de identificar a intervenção correta para contribuir no controle de riscos para o desenvolvimento de doenças cardiovasculares, ou qualquer tipo de risco relacionado ao uso contínuo da hemodiálise, também se faz necessário implementar intervenções adequadas para os pacientes cardiopatas que necessitem deste procedimento. Porém, para definir o tipo de intervenção a ser aplicada, o enfermeiro precisa seguir um modelo padrão assistencial, onde o processo de enfermagem seja implementado com o objetivo de organizar o fluxo de cuidado e padronizar a assistência de enfermagem e este modelo deve ser baseado em evidências, assim como toda a prática de enfermagem.

Ao considerar que a abordagem no atendimento ao paciente cardiopata em tratamento de hemodiálise requer um olhar diferenciado, foi identificado o seguinte problema de pesquisa: quais são os cuidados de enfermagem ao paciente com doença renal crônica cardiopata durante o procedimento de hemodiálise convencional? Dessa forma, o objetivo deste estudo foi identificar os cuidados de enfermagem ao paciente renal crônico com cardiopatia durante o procedimento de hemodiálise convencional.

\section{Metodologia}

Optou-se por uma pesquisa descritiva e qualitativa, na qual os autores trataram os dados obtidos por meio da pesquisa bibliográfica, que de acordo com Cesário, Flauzino e Mejia (2020) é direcionada para responder alguma dúvida ou questionamento, que pode incluir artigos científicos publicados em periódicos acadêmicos. Dessa forma, inicialmente buscouse reunir evidências para responder ao questionamento de pesquisa. Na etapa inicial, a busca foi realizada em bibliotecas digitais, que permitem uma busca em bases de dados próprias por meio da utilização de descritores padronizados. No DeCS (Descritores em Ciências da Saúde), foram encontrados os seguintes descritores: doença renal crônica, hemodiálise, doenças cardiovasculares, cuidados de enfermagem, que representam as strings de busca. 
Os bancos de dados utilizados foram o Google Acadêmico, BVS (biblioteca virtual em saúde) e SciELO (Scientific Electronic Library Online). No Google Acadêmico utilizou-se cada um dos descritores entre aspas (“"). Na BVS foi utilizado a opção pesquisa avançada, selecionada as bases da BDENF (Banco de Dados em Enfermagem), LILACS (Literatura LatinoAmericana e do Caribe em ciências da saúde) e o operador lógico booleano “OR” e “AND”. Na SciELO, foi utilizada a opção pesquisa avançada e o operador lógico booleano "OR" e “ $A N D$ ”.

Estabeleceu-se como critérios de inclusão: artigos acadêmicos publicados entre 2016 e 2021, na língua portuguesa, disponíveis de forma completa nos bancos de dados já mencionados e que respondessem à pergunta de pesquisa. Excluíram-se artigos repetidos encontrados nas bases de dados, resumos, artigos inferiores a 2016, artigos que não respondiam o problema da pesquisa e aqueles que não estavam publicados em revista acadêmica com ISSN (International Standard Serial Number). A coleta dos dados foi realizada no mês de março, por dois pesquisadores de forma independente. Após a captura de informações, foi utilizado um formulário de extração de dados para melhor organizar as informações que respondiam ao problema de pesquisa. Posteriormente procedeu-se a análise temática dos assuntos. Os resultados das buscas pelos dados e do número final de publicações que fizeram parte da revisão de bibliografia são apresentados na Figura 1, conforme orientações Prisma na forma de fluxograma. (Peters, 2015).

Figura 1. Fluxograma de Prisma.

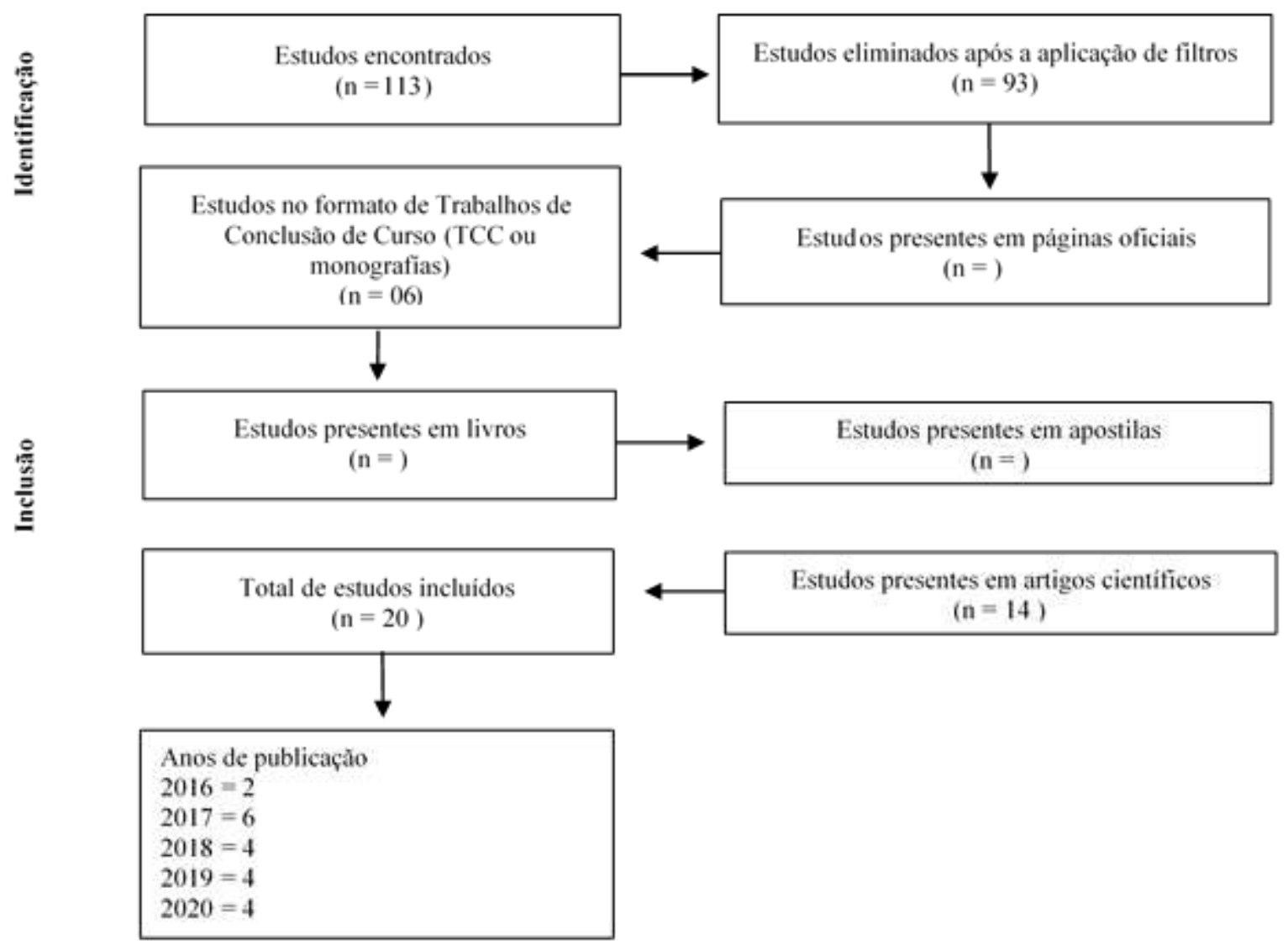

Fonte: Autores (2021). 


\section{Resultados}

A Tabela 1 foi elaborada para mostrar os artigos científicos incluídos em cada base de dados, nesta tabela é possível observar que o maior aproveitamento de artigos ocorreu na base de dados Google Acadêmico, seguida da BVS e, por fim na SciELO. A amostra final foi de 21 artigos científicos.

Tabela 1. Distribuição de artigos de acordo com Base de Dados.

\begin{tabular}{lcc} 
& \multicolumn{2}{c}{ Artigos científicos } \\
\multicolumn{1}{c}{ Bases de Dados } & Total & Incluídos \\
\hline BVS & 53 & 07 \\
ScIELO & 20 & 04 \\
Google acadêmico & 40 & 10 \\
Total & $\mathbf{1 1 3}$ & $\mathbf{2 1}$ \\
\hline
\end{tabular}

Fonte: Autores (2021).

Os resultados que foram encontrados no desenvolvimento da pesquisa de revisão bibliográfica estão representados no Quadro 1, que apresenta as seguintes variáveis: autor, título, objetivo central e tipo de estudo. Antes do início da discussão, foi elaborado o Quadro 1, para melhor apresentação dos dados encontrados, assim como para que fosse realização a comparação entre os estudos e facilitar o entendimento do leitor.

Quadro 1. Artigos incluídos neste estudo.

\begin{tabular}{|c|c|c|c|}
\hline Autor/ano & Título & Objetivos & Tipo de estudo \\
\hline Fernandes, 2016 & $\begin{array}{l}\text { Alterações cardiovasculares e } \\
\text { pulmonares em pacientes submetidos } \\
\text { à hemodiálise }\end{array}$ & $\begin{array}{l}\text { Identificar alterações cardiovasculares e } \\
\text { pulmonares em pacientes submetidos à } \\
\text { hemodiálise, correlacionando-as aos aspectos } \\
\text { socioeconômicos }\end{array}$ & Estudo transversal. \\
\hline $\begin{array}{l}\text { Marques et al., } \\
2019\end{array}$ & $\begin{array}{l}\text { Análise de indicadores de qualidade e } \\
\text { características clínicas em uma } \\
\text { unidade de terapia renal substitutiva. }\end{array}$ & $\begin{array}{l}\text { Conhecer as características sociodemográficas, } \\
\text { clínicas e indicadores de qualidade do tratamento } \\
\text { hemodialítico em unidade de terapia renal } \\
\text { substitutiva de um município brasileiro a fim de } \\
\text { que medidas estratégicas sejam realizadas para } \\
\text { otimização do cuidado destes usuários. }\end{array}$ & $\begin{array}{l}\text { Estudo quantitativo e } \\
\text { descritivo. }\end{array}$ \\
\hline $\begin{array}{l}\text { Fernandes, Enders } \\
\text { \& Lira } 2017\end{array}$ & $\begin{array}{l}\text { Análise do conceito sobrecarga de } \\
\text { líquidos em pacientes com doença } \\
\text { renal crônica em terapia dialítica: } \\
\text { revisão integrativa. }\end{array}$ & $\begin{array}{l}\text { Analisar o conceito sobrecarga de líquidos em } \\
\text { pacientes com doença renal crônica em terapia } \\
\text { dialítica e compará-lo ao diagnóstico de } \\
\text { enfermagem Volume de líquidos excessivo } \\
\text { presente na NANDA Internacional. }\end{array}$ & $\begin{array}{l}\text { Revisão integrativa da } \\
\text { literatura. }\end{array}$ \\
\hline $\begin{array}{l}\text { Carvalho et al., } \\
2020\end{array}$ & $\begin{array}{l}\text { Efeitos da doença e adequação da } \\
\text { hemodiálise em pacientes com rigidez } \\
\text { arterial }\end{array}$ & $\begin{array}{l}\text { Analisar a adequação do tratamento } \\
\text { hemodialítico em pacientes com rigidez arterial e } \\
\text { associar os efeitos da } \\
\text { doença renal com os fatores sociodemográficos e } \\
\text { clínicos. }\end{array}$ & $\begin{array}{l}\text { Estudo } \\
\text { epidemiológico } \\
\text { transversal. }\end{array}$ \\
\hline Jacon et al., 2020 & $\begin{array}{l}\text { Identificação de diagnósticos } \\
\text { enfermagem em nefropatas em } \\
\text { hemodiálise à luz da teoria das } \\
\text { necessidades humanas básicas. }\end{array}$ & $\begin{array}{l}\text { Identificar os domínios e títulos diagnósticos de } \\
\text { enfermagem recorrentes em pacientes em } \\
\text { hemodiálise, segundo a taxonomia da NANDA.I. }\end{array}$ & $\begin{array}{l}\text { Estudo descritivo e } \\
\text { transversal. }\end{array}$ \\
\hline $\begin{array}{l}\text { Moraes et al., } \\
2017\end{array}$ & $\begin{array}{l}\text { Identificação de risco cardiovascular } \\
\text { pela razão triglicerídeo/HDL- } \\
\text { colesterol em pacientes com doença } \\
\text { renal crônica em hemodiálise. }\end{array}$ & $\begin{array}{l}\text { Identificar o risco cardiovascular pela razão } \\
\text { triglicerídeo/colesterol ligado à lipoproteína de } \\
\text { alta densidade (TG/HDL-c) e verificar a sua } \\
\text { associação com fatores sociodemográficos, } \\
\text { parâmetros clínicos e índice de massa corporal } \\
\text { em pacientes com doença renal crônica em } \\
\text { hemodiálise. }\end{array}$ & $\begin{array}{l}\text { Estudo do } \\
\text { transversal. }\end{array}$ \\
\hline
\end{tabular}




\begin{tabular}{|c|c|c|c|}
\hline $\begin{array}{l}\text { Lucena et al., } \\
2017\end{array}$ & $\begin{array}{l}\text { Validação de intervenções e } \\
\text { atividades de enfermagem para } \\
\text { pacientes em terapia hemodialítica. }\end{array}$ & $\begin{array}{l}\text { Validar intervenções e atividades de enfermagem } \\
\text { propostas pela Nursing Interventions } \\
\text { Classification (NIC), para pacientes com } \\
\text { insuficiência renal aguda (IRA) ou doença renal } \\
\text { crônica agudizada, em terapia hemodialítica com } \\
\text { os diagnósticos de enfermagem (DE) Volume de } \\
\text { Líquidos Excessivo e Risco de Volume de } \\
\text { Líquidos. }\end{array}$ & $\begin{array}{l}\text { Validação } \\
\text { conteúdo }\end{array}$ \\
\hline Castro, 2018 & $\begin{array}{l}\text { Zinco e risco cardiovascular de } \\
\text { pacientes nefropatas em tratamento de } \\
\text { hemodiálise. }\end{array}$ & $\begin{array}{l}\text { Investigar a relação entre concentração de zinco } \\
\text { e risco cardiovascular em pacientes com } \\
\text { insuficiência renal crônica em hemodiálise. }\end{array}$ & $\begin{array}{l}\text { Estudo analítico, } \\
\text { retrospectivo } \\
\text { transversal. }\end{array}$ \\
\hline et al., & $\begin{array}{l}\text { Diagnósticos de enfermagem em } \\
\text { idosos com doença renal crônica em } \\
\text { hemodiálise. }\end{array}$ & $\begin{array}{l}\text { Identificar os principais diagnósticos de } \\
\text { enfermagem (DEs) em pacientes idosos em } \\
\text { tratamento hemodialítico. }\end{array}$ & Pesquisa exploratória \\
\hline $\begin{array}{l}\text { Ribeiro } \quad \& \\
\text { Andrade, } 2018\end{array}$ & $\begin{array}{l}\text { Enfermeiro protagonista na educação } \\
\text { em saúde para o autocuidado de } \\
\text { pacientes com doenças renal crônica. }\end{array}$ & $\begin{array}{l}\text { Descrever as atribuições do enfermeiro no } \\
\text { processo de educação em saúde do paciente com } \\
\text { doença renal crônica }\end{array}$ & $\begin{array}{lr}\text { Revisão } & \text { bibliográfica } \\
\text { de } & \text { abordagem } \\
\text { qualitativa }\end{array}$ \\
\hline $\begin{array}{l}\text { Guimarães et al., } \\
2018\end{array}$ & $\begin{array}{l}\text { Causas de óbito de } \\
\text { Insuficiência Renales } \\
\text { hemodiálise }\end{array}$ & $\begin{array}{l}\text { Revisar na literatura científica as causas de } \\
\text { óbitos de pacientes com insuficiência renal } \\
\text { crônica em hemodiálise }\end{array}$ & Revisão integrativa \\
\hline $\begin{array}{l}\text { Menezes et al., } \\
2020\end{array}$ & $\begin{array}{l}\text { Termos da linguagem especializada de } \\
\text { enfermagem para pacientes renais } \\
\text { crônicos em tratamento conservador. }\end{array}$ & $\begin{array}{l}\text { Validar quanto ao conteúdo termos da linguagem } \\
\text { especializada de Enfermagem utilizada no } \\
\text { cuidado às pessoas com doença renal crônica em } \\
\text { tratamento conservador identificados na } \\
\text { literatura, mapeando-os com os termos da } \\
\text { Classificação Internacional para a Prática de } \\
\text { Enfermagem, versão } 2019\end{array}$ & $\begin{array}{l}\text { Estudo descritivo, } \\
\text { documental } \\
\text { metodológico. }\end{array}$ \\
\hline $\begin{array}{l}\text { Ribeiro et al., } \\
2020\end{array}$ & $\begin{array}{l}\text { Encadeamentos da Doença Renal } \\
\text { Crônica e o impacto na qualidade de } \\
\text { vida de pacientes em hemodiálise }\end{array}$ & $\begin{array}{l}\text { Descrever os encadeamentos da Doença Renal } \\
\text { Crônica na qualidade de vida de pacientes em } \\
\text { hemodiálise; caracterizar o impacto na qualidade } \\
\text { de vida de pacientes em hemodiálise }\end{array}$ & $\begin{array}{l}\text { Pesquisa bibliográfica } \\
\text { de abordagem } \\
\text { qualitativa e caráter } \\
\text { descritivo. }\end{array}$ \\
\hline Souza et al., 2018 & $\begin{array}{llr}\begin{array}{l}\text { Assistência de enfermagem } \\
\text { crianças às }\end{array} & \text { e adolescentes } & \text { com } \\
\text { insuficiência renal crônica: } & \text { uma } \\
\text { revisão integrativa da literatura. } & \end{array}$ & $\begin{array}{l}\text { Analisar sobre a os cuidados de enfermagem às } \\
\text { crianças e adolescentes com insuficiência renal } \\
\text { crônica. }\end{array}$ & $\begin{array}{l}\text { Revisão integrativa da } \\
\text { literatura. }\end{array}$ \\
\hline $\begin{array}{l}\text { Silva \& Mattos, } \\
2019\end{array}$ & $\begin{array}{l}\text { Complicações hemodialíticas } \\
\text { Unidade de Terapia Intensiva. }\end{array}$ & $\begin{array}{lll}\text { Caracterizar } & \text { o perfil sociodemográfico e } \\
\text { identificar complicações em pacientes } \\
\text { submetidos ao tratamento hemodialítico } \\
\text { internados em Unidade de Terapia Intensiva. }\end{array}$ & $\begin{array}{l}\text { Estudo quantitativo, } \\
\text { transversal } \\
\text { descritivo. }\end{array}$ \\
\hline $\begin{array}{l}\text { Oliveira et al., } \\
2019\end{array}$ & $\begin{array}{l}\text { tuação do enfermeiro na prevenção } \\
\text { e doença renal crônica em portadores } \\
\text { e diabetes: revisão integrativa. }\end{array}$ & $\begin{array}{l}\text { Relacionar a atuação do enfermeiro na prevenção } \\
\text { de doença renal crônica em portadores de } \\
\text { diabetes. }\end{array}$ & $\begin{array}{l}\text { Revisão integrativa da } \\
\text { literatura. }\end{array}$ \\
\hline $\begin{array}{l}\text { Freitas et al., } \\
2018\end{array}$ & $\begin{array}{l}\text { Assistência de enfermagem visando a } \\
\text { qualidade de vida dos pacientes renais } \\
\text { crônicos na hemodiálise }\end{array}$ & $\begin{array}{l}\text { Compreender a importância da assistência de } \\
\text { enfermagem, voltada a qualidade de vida do } \\
\text { paciente renal crônico. }\end{array}$ & $\begin{array}{l}\text { Trata-se de uma } \\
\text { revisão da literatura } \\
\text { para levantamento dos } \\
\text { artigos. }\end{array}$ \\
\hline $\begin{array}{l}\text { Spigolon et al., } \\
2018\end{array}$ & $\begin{array}{l}\text { Diagnósticos de enfermagem de } \\
\text { portadores de doença renal em } \\
\text { hemodiálise: estudo transversal }\end{array}$ & $\begin{array}{l}\text { Identificar os Diagnósticos de Enfermagem de } \\
\text { portadores de doença renal crônica em estádio } 5 \\
\text { hemodialítico }\end{array}$ & $\begin{array}{l}\text { Estudo transversal, } \\
\text { descritivo, de } \\
\text { abordagem } \\
\text { quantitativa }\end{array}$ \\
\hline $\begin{array}{l}\text { Almeida et al., } \\
2019\end{array}$ & $\begin{array}{l}\text { Envolvimento da pessoa com doença } \\
\text { renal crônica em seus cuidados: } \\
\text { revisão integrativa. }\end{array}$ & $\begin{array}{l}\text { Descrever o manejo a doença renal e seu } \\
\text { tratamento. }\end{array}$ & Revisão integrativa \\
\hline $\begin{array}{l}\text { Xavier et al., } \\
2018\end{array}$ & $\begin{array}{l}\text { Na correnteza da vida: a descoberta da } \\
\text { doença renal crônica. }\end{array}$ & $\begin{array}{l}\text { Reconstruir, a partir da escuta, a trajetória de } \\
\text { vida de uma paciente renal crônica em } \\
\text { tratamento hemodialítico. }\end{array}$ & Pesquisa qualitativa \\
\hline
\end{tabular}




\section{Discussão}

Para que à assistência de enfermagem seja realizada de forma adequada, é necessário a padronização do processo de enfermagem (PE) com o objetivo de aplicar os conhecimentos técnico-científicos e assim, realizar a prática profissional de forma que garanta a segurança do paciente e que os objetivos terapêuticos sejam alcançados. O PE é composto por cinco etapas: histórico, diagnóstico, planejamento, intervenção e avaliação. As intervenções de enfermagem individualizadas são realizadas, primeiramente por meio dos diagnósticos de enfermagem (DE), que é o pilar de essencial de todo o processo de enfermagem e definirá as intervenções de enfermagem específicas para cada paciente, baseadas em suas necessidades reais. Para isso, são utilizados alguns sistemas de classificação, para que a enfermagem empregue uma linguagem única, objetiva e clara. Os sistemas de classificação mais comuns são a North American Nursing Diagnosis Association (NANDA), a Classificação das Intervenções de Enfermagem (NIC), Classificação dos Resultados de Enfermagem (NOC) e a Classificação Internacional para a Prática da Enfermagem (CIPE) (Debone et al., 2017). O NANDA classifica os DE, o NIC classifica as intervenções, relacionadas aos DE e o NOC classifica os resultados esperados e é relacionado aos Des. O CIPE é outra classificação utilizada, que relaciona os diagnósticos com as intervenções de enfermagem.

A implementação do processo de enfermagem auxilia de maneira significativa a sistematização da assistência de enfermagem (SAE). No momento em que o enfermeiro percebe o paciente como um todo, com uma visão holística, ele consegue identificar suas necessidades e elabora um plano de cuidados completo e personalizado. Para iniciar corretamente o $\mathrm{PE}$, é importante que o enfermeiro faça a coleta de dados e aplique os diagnósticos adequadamente. O planejamento da assistência deve estar direcionado aos principais riscos encontrados e os cuidados prescritos devem ser individualizados, com o objetivo de atuar na prevenção e na promoção à saúde, bem como incentivar a aderência ao tratamento (Spigolon et al. 2018).

Os DEs são relevantes, pois são ferramentas imprescindíveis no atendimento não somente a pacientes cardiopatas em terapia hemodialítica, mas a qualquer paciente que necessite da assistência de enfermagem. Diagnósticos bem definidos e estabelecidos podem melhorar muito a qualidade no atendimento a estes pacientes. De acordo com Jacon et al. (2020), foram encontrados 984 DE diferentes em pacientes dialíticos, mais da metade possui risco, com prevalência no risco de infecção, enquanto os demais estavam focados no problema, na maioria, estilo de vida sedentária. Os autores afirmam, ainda, que elaborar um plano terapêutico pautado na taxonomia NANDA traz impacto relevante na qualidade de vida do paciente em hemodiálise e maior domínio para planejar ações e intervenções resolutivas.

De acordo com Freitas et al. (2018), os DE mais prevalentes e encontrados em pacientes cardiopatas em hemodiálise foram risco de desequilíbrio eletrolítico, pela disfunção renal inerente aos pacientes renais crônicos; risco de perfusão renal ineficaz, associado à má aderência ao regime terapêutico; risco de resposta adversa a meio de contraste com iodo, que ocorre devido a uma desidratação provocada pelo iodo utilizado em alguns meio de contraste durante exames ou procedimentos; risco de infecção, relacionado a procedimentos invasivos, como punção da fístula arteriovenosa (FAV) ou presença de cateter duplo lúmen (CDL); risco de trauma vascular, identificado devido a problemas causados pela presença de CDL, como hematomas, período de permanência ou estenose central, ou comprometimento vascular por cuidados inadequados com a FAV e eliminação urinária prejudicada, ocasionada pela redução da taxa de filtração glomerular, que pode levar a uma alteração na produção urinária.

O DE volume de líquidos excessivo definido pela taxonomia de NANDA como retenção aumentada de líquidos isotônicos é semelhante ao conceito "sobrecarga de líquidos em pacientes hemodialíticos". Estudos realizados por Fernandes, Enders e Lira (2017) comprovaram que deve ser feita uma melhoria dos componentes do DE volume de líquidos excessivo da NANDA para que seja capaz de dar seguimento ao paciente dialítico. No entanto, a dificuldade de seguimento de restrição de fluidos e na dieta também foi prevalente, embora não esteja em NANDA. Foi relatado entre as principais consequências da 
sobrecarga de líquidos em pacientes dialíticos o ganho de peso e aumento da pressão arterial e consequentemente maior risco de doenças cardiovasculares.

Quanto ao volume de líquidos excessivo e risco de volume de líquidos desequilibrados, Lucena et al. (2017) afirmam que estes correspondem aos DE mais estabelecidos durante o plano de enfermagem do paciente dialítico. A NIC classifica como intervenções para os DE estabelecidos: controle hídrico, controle da hipervolemia, monitoração hídrica e monitoração de eletrólitos. Os autores ressaltam que a intervenção NIC considerada prioritária para os profissionais em ambos DE foi o controle hídrico. As atividades de enfermagem consideradas prioritárias, apresentam-se em comum para $6 \mathrm{DE}$, o que confirma o direcionamento do cuidado para esses pacientes, que contribui na elaboração de um plano de cuidado voltado às reais necessidades do doente renal crônico em terapia hemodialítica.

O ponto chave para entender o porquê o diagnóstico de enfermagem é tão importante, é que as intervenções são baseadas nos diagnósticos individuais de cada paciente, e a execução dessas intervenções faz-se imprescindível para resultados que contribuem para uma melhor qualidade de vida e ganhos na saúde em geral (Debone et al., 2017). De acordo com Ribeiro \& Andrade (2018), os cuidados considerados mais importantes pelos enfermeiros foram os cuidados com o acesso vascular bem como as necessidades de segurança e educação em saúde. Além disso, a mobilização e orientações sobre as restrições dietéticas também se mostraram relevantes. O enfermeiro contribui na adaptação dos pacientes à sua nova realidade por meio da educação em saúde, com a consequente melhora da compreensão e adesão ao tratamento. O enfermeiro colabora também para que os pacientes desenvolvam autoconhecimento e liderem seu processo de doença. É essencial que os cuidados realizados sigam uma padronização para que sejam mais eficazes, ao mesmo tempo em que precisam atender às necessidades específicas de cada paciente.

Segundo Souza et al. (2018), a complicação mais comum entre pacientes em terapia hemodialítica é a hipotensão, que pode acontecer devido a uma alta taxa de ultrafiltração ou alta temperatura do dialisato, entre outras causas. Para prevenir a hipotensão, é necessário um controle rigoroso dos sinais vitais e observação de sinais e sintomas que indiquem queda na pressão arterial. Caso ocorra, deve-se colocar o paciente na posição de Trendelemburg, administrar soro fisiológico a 0,9\% e ajustar a taxa de ultrafiltração para uma menor perda por hora. Para uma melhor assistência frente às intercorrências, os profissionais devem estar sempre atualizados quanto às intervenções e cuidados que devem ser realizados, avaliar as necessidades individuais e evolução destes pacientes, de modo a prestar um atendimento seguro e de qualidade.

Menezes et al. (2020) salientaram a importância da inserção dos pacientes em terapia hemodialítica na construção de sua autonomia, potencialmente limitada pela hemodiálise. Foi mencionado pelos autores a importância do Enfermeiro Especialista em Reabilitação (EER), que é o responsável por elaborar e implementar programas de cuidados especializados, com o intuito de ajudar estes pacientes que estão incapacitados de realizar suas atividades de vida diárias (AVD), eles definem o plano de exercícios intra-dialíticos mais adequado que auxilie na prevenção e reabilitação para o doente renal crônico dialítico. Este plano de exercícios, considerado prescrição terapêutica, visa promover a independência e autonomia destes pacientes, a fim de manter ou recuperar suas capacidades funcionais. Dessa forma, observa-se que a intervenção do EER com programas de exercícios físicos intra-dialíticos interferiu positivamente na capacidade funcional e na percepção da capacidade física na realização das AVD, mesmo com as limitações e restrições apresentadas por estes pacientes. Desta forma, eles podem viver de forma mais independente, com autonomia para melhorar o autocuidado.

Os cuidados com o acesso vascular foram mencionados por Oliveira et al. (2019) como intervenções fundamentais. Geralmente, pacientes hospitalizados com lesões renais agudas (LRA) fazem uso de cateter temporário duplo-lúmen em veia central e profunda, preferencialmente a veia jugular interna direita. É função da enfermagem manter este acesso pérvio, por meio da infusão de solução fisiológica a $0,9 \%$ e selagem com trombolíticos, a fim de minimizar os riscos de obstrução. Associado a isso, a utilização de técnica asséptica na realização do curativo oclusivo e na manipulação do cateter durante as 
sessões previne a colonização do dispositivo e possíveis infeções na corrente sanguínea. Vale ressaltar que todas as complicações e procedimentos devem ser devidamente registrados em prontuário para que haja um melhor acompanhamento e maior qualidade na assistência prestada ao paciente com LRA.

O profissional enfermeiro está inserido no contexto que se resume a proporcionar a assistência adequada, e a atenção integral e humanizada à saúde do paciente. É levado em consideração que o enfermeiro é considerado um educador e que deve colaborar e estimular a adesão ao tratamento. Portanto, faz-se necessária a criação de um vínculo de confiança entre ele, paciente e a família para esclarecimento de dúvidas e ajuda na adaptação ao novo estilo de vida. Estabelecer estas relações interpessoais e agir com empatia para com os pacientes, é a garantia de oferecer um cuidado humanizado, a fim de propiciar condições favoráveis ao tratamento (Ribeiro, et al. 2020).

Portanto, a evolução do processo de enfermagem no planejamento da assistência do paciente garante uma padronização da linguagem e dos cuidados dos profissionais da área e aprimora esse cuidado, ao alicerçá-lo em bases mais cientificas e menos indutivas. A enfermagem atua diretamente no processo de recuperação e reabilitação do paciente por meio do plano de intervenções e é por esse motivo, que ao tratar um paciente renal crônico com cardiopatia em hemodiálise, o enfermeiro necessita, além de uma intervenção direta, planejar uma assistência baseada em uma abordagem humanizada e eficientes, onde sejam claras as reais necessidades desse paciente crônicos, que na maioria das vezes, precisam conviver com esses tipos de doença para o resto da vida.

\section{Considerações Finais}

O cuidado de enfermagem ao paciente renal crônico com cardiopatia em hemodiálise requer o desenvolvimento de práticas específicas que atenda as suas reais necessidades. O foco do cuidado do enfermeiro está em promover a saúde e prevenir a doença, porém, grande é o desafio para a enfermagem em prevenir as complicações oriundas dessa doença. O enfermeiro precisa oferecer um cuidado padronizado, de forma que melhor organize os serviços de enfermagem, além de, facilitar e profissionalizar ainda mais a linguagem utilizada na área. Observou-se então, que essa forma de padronização se dá por meio da implementação do processo de enfermagem, o qual, a partir dos diagnósticos estabelecidos, determina e organiza as intervenções realizadas pela equipe de enfermagem.

Apesar do grande desafio encontrado pelos enfermeiros em realizar os cuidados necessários em relação à pacientes dialíticos com cardiopatia, manter o acesso vascular, educação em saúde, segurança, mobilização e dieta, são os cuidados mais relevantes considerados pelos enfermeiros, para esses pacientes. Em relação a colaboração e função do enfermeiro no processo saúde-doença, é primordial que a enfermagem ajude esse paciente a se adaptar à uma nova realidade, ao prestar um cuidado humanizado, onde visa paciente, ambiente onde esse indivíduo convive, e família. Nesse contexto, o cuidado com o estado psicológico do doente é um outro ponto relevante, onde o enfermeiro precisa orientar o paciente para que ele entenda e lidere o processo da doença.

Portanto, o aprimoramento das práticas de enfermagem, assim como, a conscientização para da importância da correta aplicação do processo de enfermagem é de fundamental importância, pois colabora também para que haja a garantia de uma prática de qualidade e segura. Espera-se com este estudo estimular a discussão a respeito da assistência de enfermagem ao paciente renal crônico com cardiopatia em hemodiálise, pois ainda há uma escassez de estudos em português a respeito deste tema.

\section{Referências}

Almeida, O. A. E., et al. (2019). Envolvimento da pessoa com doença renal crônica em seus cuidados: revisão integrativa. Ciência \& Saúde Coletiva. 24(5). 1689-1698. http://dx.doi.org/10.1590/1413-81232018245.04332019. 
Carvalho, L., et al. (2020). Efeitos da doença e adequação da hemodiálise em pacientes com rigidez arterial. Escola Anna Nery. 24(2). http://dx.doi.org/10.1590/2177-9465-ean-2019-0068.

Castro, Á. S. (2018). Zinco e risco cardiovascular de pacientes nefropatas em tratamento de hemodiálise. Revista Brasileira em Promoção da Saúde. 31(1). http://dx.doi.org/10.5020/18061230.2018.5803.

Cesário, J. M. S., Flauzino, V. H. P \& Mejia, J. V. C. Metodologia científica: Principais tipos de pesquisas e suas caraterísticas. Revista Científica Multidisciplinar Núcleo do Conhecimento. 5(11)23-33. http://dx.doi.org/10.32749/nucleodoconhecimento.com.br/educacao/tipos-de-pesquisas.

Debone, M. C. (2017). Diagnósticos de enfermagem em idosos com doença renal crônica em hemodiálise. Revista Brasileira Enfermagem. 70(4):800-5. http://dx.doi.org/10.1590/0034-7167-2017-0117.

Fernandes, M. I. C. D., Enders, B. C. \& Lira, A. L. B. C. Análise do conceito sobrecarga de líquidos em pacientes com doença renal crônica em terapia dialítica: revisão integrativa. Revista escola de enfermagem (USP). 1(51). e03299. http://dx.doi.org/10.1590/s1980-220x2016036003299.

Fernandes, M. I. C. D., et al. (2016). Alterações cardiovasculares e pulmonares em pacientes submetidos à hemodiálise. Revista Enfermagem UERJ. 24(3). e8634. http://dx.doi.org/10.12957/reuerj.2016.8634.

Freitas E. A. (2018). Assistência de enfermagem visando a qualidade de vida dos pacientes renais crônicos na hemodiálise. Revista de Iniciação Científica e Extensão. 1(2). 114-21.

Guimarães, D. B. O et al. (2018). Causas de óbito de pacientes com Insuficiência Renal Crônica em hemodiálise. Revista Eletrônica Acervo Saúde. 10(3). 1595-1604. http://dx.doi.org/10.25248/REAS159_2018.

Jacon, J. C., et al. (2020). Identificação de diagnósticos de enfermagem em nefropatas em hemodiálise à luz da teoria das necessidades humanas básicas. Revista CuidArte Enfermagem. 14(1). 48-54

Lucena, A. F., et al. (2017). Validação de intervenções e atividades de enfermagem para pacientes em terapia hemodialítica. Revista Gaúcha Enfermagem. 38(3). e66789. http://dx.doi.org/10.1590/1983-1447.2017.03.66789

Matos, G. C., et al. (2020). Avaliação da fragilidade de doentes renais crônicos em tratamento de hemodiálise. Revista Contexto \& Saúde. 20(38).28-33. http://dx.doi.org/10.21527/2176-7114.2020.38.28-33.

Marques, B. M., et al. (2019). Análise de indicadores de qualidade e características clínicas em uma unidade de terapia renal substitutiva. Revista CuidArte Enfermagem. 13(2). 99-105.

Menezes, H. F., et al. (2020). Termos da linguagem especializada de enfermagem para pacientes renais crônicos em tratamento conservador. Revista Brasileira de Enfermagem. 73(6). e20190820. http://dx.doi.org/10.1590/0034-7167-2019-0820.

Moraes, L. L., et al. (2017). Identificação de risco cardiovascular pela razão triglicerídeo/HDL-colesterol em pacientes com doença renal crônica em hemodiálise. Revista Scientia Medica. 27(3). http://dx.doi.org/10.15448/1980-6108.2017.3.27369.

Neves, P. D. M. D. M., et al. (2020). Censo Brasileiro de Diálise: análise de dados da década 2009-2018. Brazilian Journal of Nephrology, 42(2), 191-200.

Oliveira, F. J. S. et al (2019). Atuação do enfermeiro na prevenção de doença renal crônica em portadores de diabetes: revisão integrativa. Revista Eletrônica Acervo Saúde. 1(30). e927. http://dx.doi.org/10.25248/reas.e927.2019.

Peters, M. D., et al. (2015). Guidance for conducting systematic scoping reviews. International Journal of Evidence-Based Healthcare. 13(3). 141-146. http://dx.doi.org/10.1097/XEB.0000000000000050.

Ribeiro, W. A., et al. (2020). Encadeamentos da doença renal crônica e o impacto na qualidade de vida de pacientes em hemodiálise. Revista Pró-Univer SUS 11(2). 111-120.

Santos, K. A. S., et al. (2021). Principais intercorrências durante sessões de hemodiálise em pacientes com comorbidades. Brazilian Journal of Development. 7(2). 14066-14079. http://dx.doi.org/10.34117/bjdv7n2-162

Silva, M. R., et al. (2020). Qualidade de vida de pacientes renais crônicos submetidos á hemodiálise: Uma revisão integrativa. Brazilian Journal of Health Review. 3(4). 9344-9374. http://dx.doi.org/10.34119/bjhrv3n4-172.

Ribeiro, W. A., et al. (2020). Encadeamentos da Doença Renal Crônica e o impacto na qualidade de vida de pacientes em hemodiálise. Revista PróUniverSUS. 11(2). (111-120).

Ribeiro, W.A., \& Andrade, M. (2018). Enfermeiro protagonista na educação em saúde para o autocuidado de pacientes com doenças renal crônica. Revista Pró-UniverSUS. 09 (2): 60-65.

Silva, P. E. B. B., \& Mattos, M. (2019). Complicações hemodialíticas na Unidade de Terapia Intensiva. Revista de Enfermagem UFPE online. 13(1). 162-168.

Sociedade Brasileira de Nefrologia. Doença renal crônica: diagnóstico e prevenção. https://www.sbn.org.br/noticias/single/news/doenca-renal-cronicadiagnostico-e-prevencao/

Spigolon, D. N et al. (2018). Diagnósticos de enfermagem de portadores de doença renal em hemodiálise: estudo transversal. Revista Brasileira de Enfermagem. 71(4). 2014-2020. http://dx.doi.org/10.1590/0034-7167-2017-0225.

Souza D. G., et al. (2018). Assistência de enfermagem às crianças e adolescentes com insuficiência renal crônica: uma revisão integrativa da literatura. Revista Remecs. 3(5). 28-37.

Xavier, S. S. M., et al. (2018). Na correnteza da vida: a descoberta da doença renal crônica. Revista Interface. 22(66). 841-51. http://dx.doi.org/10.1590/180757622016.0834 . 